\title{
A Comparison Between Geometric and Bio-Inspired Algorithms for Solving Routing Problem in Wireless Sensor Network
}

\author{
Karima Aksa ${ }^{1, *}$, Mohammed Benmohammed ${ }^{2}$ \\ ${ }^{1}$ Department of computer science, El hadj lakhdar University, Batna, 05000, Algeria \\ ${ }^{2}$ Department of computer science, Mentouri University, Constantine, 25000, Algeria
}

\begin{abstract}
One of the important research topics in the literature of Wireless Sensor Network (WSN) is the design of an efficient routing protocol. This latter is a continuous challenging task due to the networks' variable topology and limited resources. Many researchers have proposed various routing protocols for WSN using some principles taken from geometric field or modifying some classical protocols or inspiring algorithms from nature (bio-inspired system). In this paper, we have tried to make a comparison between geometric field and bio-inspired system through a case study of two algorithms: one, which has already existed, known as DIR or compass routing protocol and the other one, which is a new proposal, known as BeeRP (BeeRoutingProtocol) inspired from bees' communication. The outcome of this work shows that both algorithms are similar having the same intrinsic principle based on direction towards the destination in routing, whereas their tools differ. Therefore; the choice of nature, with its boundless source of ideas, proves that it can give the same solutions as geometric field.
\end{abstract}

Keywords Wireless Sensor Network, Routing Protocol, Bio-Inspired System

\section{Introduction}

Wireless sensor networks (WSN) have been identified as being key technology in monitoring and detecting threats. They consist of a set of nodes powered by batteries and collaborating to perform sensing tasks in a given environment. They have a wide range of applications such as environmental monitoring, biomedical research, human imaging and tracking, and military applications[2,5].

Wireless sensor network design is influenced by many factors, which include fault tolerance, scalability, production costs, operating environment, sensor network topology, hardware constraints, transmission media and power consumption.

Most of the routing algorithms for traditional networks are address centric, and the ad hoc nature of wireless sensor network makes them unsuitable for practical applications. Also the algorithms designed for mobile ad hoc networks are unsuitable for wireless sensor networks due to the severe energy constraints that require nodes to perform for months with limited resources, as well as the low data rate which the constraint implies[1].

* Corresponding author:

Karima.aksa@univ-batna.dz (Karima Aksa)

Published online at http://journal.sapub.org/ijnc

Copyright (C) 2012 Scientific \& Academic Publishing. All Rights Reserved
In general, two distinct classes of routing protocols are presented in the literature; the first one is the classical non-geographic routing protocols being either proactive, reactive or hybrid. This class suffers from a huge amount of overhead for route setup and maintenance due to the frequent topology changes; moreover the route discovery or link state updates affect negatively the traffic network and limit its scalability and efficiency. The second class is reserved for geographic routing (called geo-routing) protocols. They are very efficient due to their ability to find new routes in both static and mobile networks with frequent topology changes by using only local topology information. In this kind of protocols, nodes need to know their geographic locations (using some localization mechanisms like GPS), destination location and direct neighbors' locations.

A third issue to propose new solutions for the above problems in routing, for WSN, is entirely different, it is that of nature. Many researchers have turned the ruder towards the nature field to inspire biological ideas. They have argued $[9,10]$ that there is a great opportunity to find solutions in biology that can be applied to problems in networking[11] such as ant colony. In fact, the relationship between computer systems and biology is not a new idea[8], however, the unprecedented complexity and scale of modern networks demands investigation from a different angle in this field.

Following the path of the naturalist researchers, we have tried to compare a routing protocol inspired from bee's 
communication, from which the name BeeRP is inspired, with another routing protocol taken from the geometry field called DIR or Compass routing. The outcome of this work shows that both algorithms are similar having the same intrinsic principle based on direction towards the destination in routing, whereas their tools differ.

This paper is organized as follows. In section 2, we have given an overview about routing in wireless sensor network. Section 3 is a presentation of Geometric routing protocols for wireless sensor network. The following section (section 4) is divided into two parts; the first one is a concise presentation of the bio-inspired systems in WSN. The second part is a presentation of BeeRP (Bee Routing Protocol), a routing algorithm inspired from bee's communication. Section 5 is dedicated to the simulation to prove the similarity of the two algorithms mentioned previously. Finally, section 6 concludes the essential points and main results reached through this work along with some guided perspectives.

\section{Routing in Wireless Sensor Network}

Wireless sensor networks can be considered as ad-hoc networks. However, ad-hoc protocols do not respect some of the sensor networks requirements: sensors present low power battery, low memory, the routing tables grow up with the network length and do not support diffusion communication. These are the main reasons why it is necessary to design new protocols, built on the most important criterion energy/efficiency.

Designing an algorithm to implement the shortest path routing between nodes for information transfer is a very complex matter. The goal of any algorithm is to efficiently find a path from one node to another using the least amount of "hops" possible in a Multi-hop Wireless Network. There are many things to take into consideration before a shortest path routing algorithm can function. Power conservation and memory management create complexities that make devising an efficient route selection algorithm rather difficult. However, the research presented implements a possible solution[6].

Routing algorithms in WSNs must be designed keeping the following requirements and constraints in mind:

- Power efficiency is the most important consideration due to the limited capacity of a sensor node.

- The WSN has to be self-organising.

- WSNs are mostly data-centric.

- Position awareness is extremely important in many WSN applications.

- Data collected by sensor nodes may contain large amounts of redundancy. Therefore, in-network aggregation would have to be performed.

- The large numbers of sensor nodes in typical WSN deployments make it impossible to build a global addressing scheme.

- Post-deployment, WSN nodes are stationary in most cases. In some applications, however, sensor networks may be allowed to move and change their location (although with low mobility).

- Sensor nodes mainly use a broadcast communication paradigm, whereas most ad hoc network routing algorithms use the point-to-point communication paradigm.

Routing protocols specifically designed for sensor networks are categorized into three types: data-centric, hierarchical, and location-based. In addition, slightly different approaches such as network flow and Quality of Service (QoS) are explored to consider end-to-end delay and energy efficiency while finding paths in the wireless sensor networks.

\section{Geometric Routing Protocols for Wireless Sensor Network}

As mentioned above, geographic routing is receiving more and more attention since it is a memory-less and scalable approach. Thus many proposals and protocols have mushroomed in this field almost all over the world. From 1984 to 1986, three progress based geo-routing protocols have been gaining importance, were already proposed for packet radio networks and were lately rediscovered for mobile ad-hoc and sensor networks. These approaches are based on the progress which is defined as the projection of the distance traveled over the last hop from $S$ (source) to any node A onto the line from $S$ to the final destination D. MFR (Most Forward within Radius) proposed by Takagi and Kleinrock[1] was the first geographic routing algorithm and the first protocol using this system and. It was introduced as an attempt trying to minimize the number of hops by selecting the node with the largest progress from its neighbors. The objective of this protocol was to obtain the optimum transmission radius in a contention-based channel. Three months later, a new protocol called RPM (Random Progress Method) was proposed by Nelson and Kleinrock[2]. In fact, it is based on the MFR principle with a slight modification. In RPM packets destined towards D are routed with equal probability towards one intermediate neighboring node that has a positive progress. The rationale for the method is that; if all nodes are sending packets frequently, probability of collision grows with the distance between the nodes, and thus there is a trade-off between the progress and transmission success. Two years later, Hou and Li[3] have proposed NFP (Nearest Forward Progress) which contributed in minimizing the interference with other nodes and the overall power consumption by transmitting the packet to the nearest node with forward progress. MFR, RPM and NFP, though they are simple localized algorithms, highly efficient in a dense graph and loop-free, are nevertheless, incapable to guarantee delivery, whenever there exist voids in a network.

In 1987 Finn, in[4], had proposed a localized greedy scheme called GF (Greedy Forwarding) as variant of random progress method, where the node, currently holding the message, will forward it to the neighbor that is closest to 
destination. Only nodes closer to destination than the current node are considered. This greedy algorithm is greedy forwarding with limited flooding; and therefore; it has the same advantages and insufficiencies as the precedent algorithms.

In 1998 and 1999 was born a type of routing protocols called directional routing protocols. Two of its protocols Dream[5] and LAR[6], appeared in the same year. Dream (Distance Routing Effect Algorithm for Mobility) was proposed to use the location information for data delivery in ad hoc network. In this routing protocol the packet is forwarded to all nodes in the direction of the destination. Based on the destination location and its velocity, the source determines an expected zone for the destination and forwards the packet to all nodes within an angle containing the expected zone. If the sender has no neighbors in the direction of the destination, a recovery procedure using partial flooding or flooding is invoked. LAR (Location Aided Routing) uses the location information of nodes for route discovery and not for data delivery. It improves the performance of non-geographic ad hoc routing protocols by limiting discovery floods to a geographic area around the destination expected location. One year later, Kranakis, Singh and Urrutia have proposed in[14] DIR (DIRectional) or Compass routing where a node forwards the packet to the neighbor whose edge has the closest slope to the line between that node and the destination; that is the neighbor with the closest direction to the destination (see figure 1). For example, in figure 2, the source or intermediate node $\mathrm{A}$ uses the location information of the destination $\mathrm{D}$ to calculate its direction. Then the message $m$ is forwarded to the neighbor $\mathrm{C}$, such that the direction $\mathrm{AC}$ closest to the direction $\mathrm{AD}$. This process repeats until the destination is, eventually, reached. In this example, the path selected by DIR method is SACJKLMND.

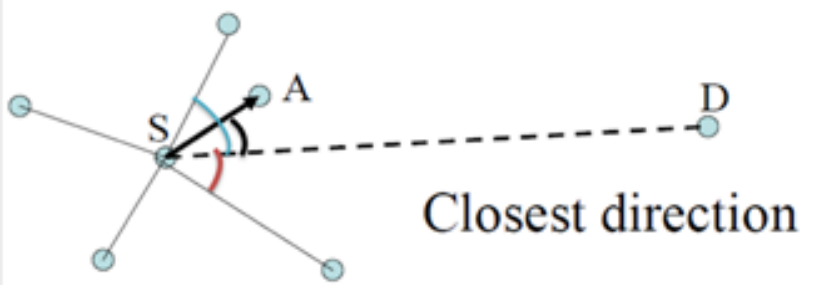

Figure 1. S selects the node A which has the smallest angle $A \hat{S} D$

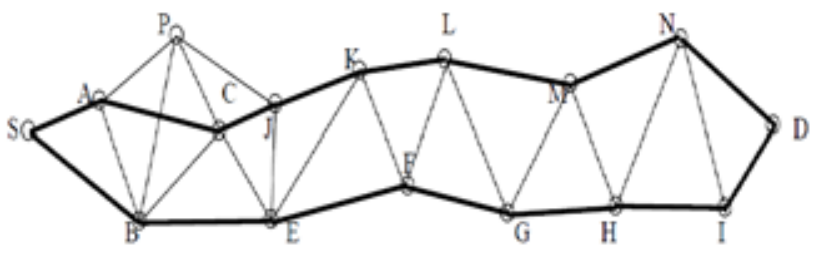

Figure 2. Using DIR protocol, the path from $\mathrm{S}$ to D is SACJKLMND

We notice that all these algorithms based directional have very high delivery rates for dense networks, but low for spars graphs. In addition to that the compass routing is not a loop-free. Another local algorithm called Compass Routing II appeared in[8], to mark its contribution in confirming the attainment of the packet to its right destination. Compass Routing II, which becomes known as face routing or pe- rimeter routing, works in planar unit graphs by traversing the faces intersecting the line between the source and the destination consecutively until reaching the destination. This algorithm was considered as the First correct algorithm.

\section{A Novel Bio-inspired Idea to Present the Same Geometric Routing Protocol for WSN: BeeRP (Bee Routing Protocol)}

The idea of bees approach is applied by several researchers to solve different problems in Network communication. There are recent works on Bee-based routing like QoS Swarm Bee Routing Protocol for Vehicular Ad Hoc, Networks, localization of wireless sensor network using Bees Optimization Algorithm and cluster based wireless sensor network routings using Artificial Bee Colony Algorithm[3-5,13].

In our turn, we have tried to take a global idea inspired from bee's communication which is the direction to reveal the existence of the geometric routing protocol called DIR[14] in the bio-inspired field.

In this section, an overview will be given about the wonderful bees' communication. The next step will be that of selecting the architecture of sensor network to apply the bio-inspired routing that we have named BeeRP. And the last step in this section will present in detail this algorithm.

\subsection{Bees' Communication}

Honeybees communicate through their movements. They attract the attention of other bees and let them know where to find nectar using movements that look like a dance. The movements show the other bees the way and the distance to reach their goal. Bees usually move in the form of figure eight. Slow dancing means the nectar is far away, fast dancing means it is nearby.

If the dance floor is horizontal, the indication of direction is straight-forward: the wagging (straight) portion of the eight-figure dance points towards the food source (and in the same direction as the bee runs through it). But, what does the dancing bee use as compass to accurately point in the right direction? The bee reference is the direction of the sun. If the dance floor is vertical, the indication of direction requires a higher-level language that can communicate horizontal directions with an indirect, symbolic, representation. In a vertical plane, the natural reference is gravity, so the dancer replaces the real reference, the sun, by the "UP" direction. For example, if the bee maintained the sun 70 degrees to her left when flying towards the nectar, the wagging portion of her dance will point 70 degrees in the clockwise direction from the upwards vertical direction. The bee transposes the solar angle into a gravitational angle! On an oblique comb the gravitational transposition works well up to an angle of about 10 degrees to the horizontal. 


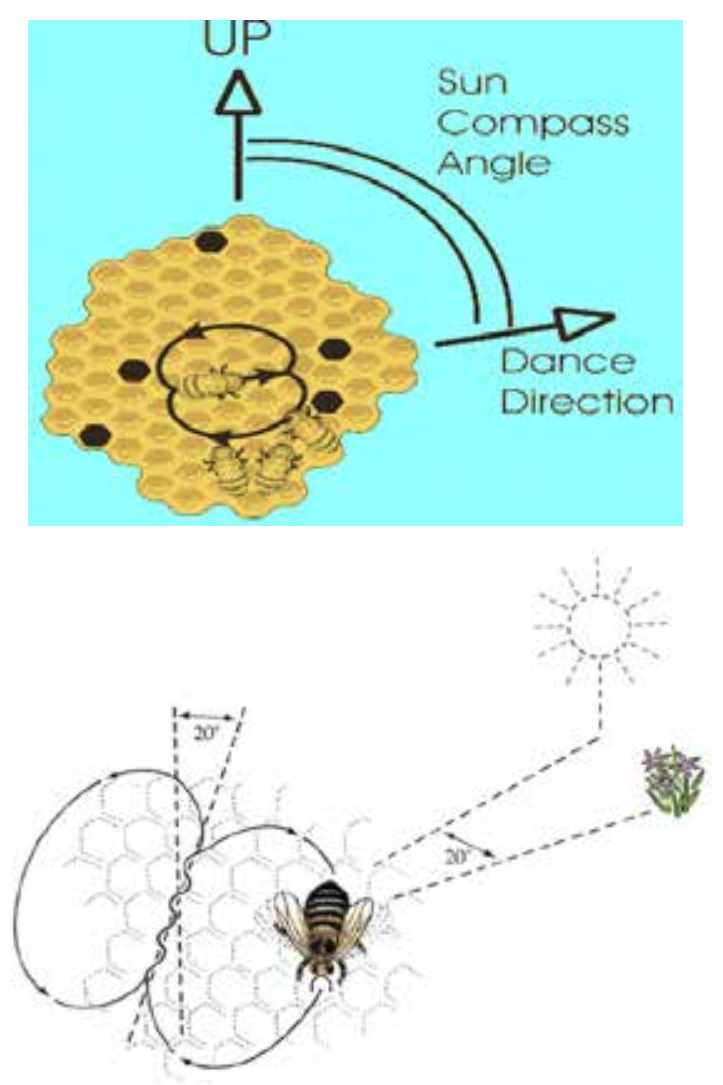

Figure 3. Bees' dance

There are two main hypotheses to explain how foragers recruit other workers - the "waggle dance" or "dance language" theory and the "odor plume" theory. The dance language theory is far more widely accepted, and has far more empirical support. The theories also differ in that the former allows for an important role of odor in recruitment (i.e., effective recruitment relies on dance plus odor), while the latter claims that the dance is essentially irrelevant (recruitment relies on odor alone). For more information, you can laminate the book called "The Dance Language and Orientation of Bees"[12]

Through bees' communication (dance), we can understand that there are three important things to learn: distance (dance's type), direction (using sun or gravity reference as a compass), and identifier 'ID' (nectar's odor for better food localization).

\subsection{The Selected Architecture to Apply Beerp Algorithm}

Because of the scarce energy supply of a wireless sensor network, the task of choosing network architecture becomes a prerequisite to optimize the energy consumption. Since, in a multi-hop network, a sensor spends most of its energy in relaying data packet. It is important to shorten the distance spent by the packet until reaching the sink. These distances can be reduced seriously by fixing a single sink in the center of the sensor field as it is illustrated in figure 3. Due to the efficiency of this architecture, several researchers have selected them like[7].

For this most reason (optimizing the energy consumption), we have chosen this kind of architecture to apply BeeRP.

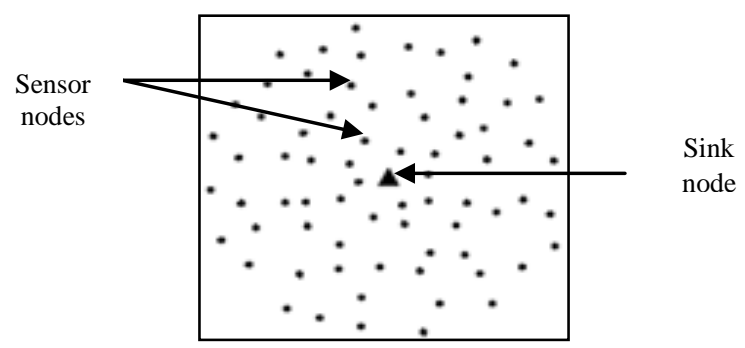

Figure 4. Architecture of sensor network with a central sink

\section{3. "BeeRP” Geographic Routing Protocol for Wireless Sensor Network}

BeeRP "Bee routing protocol" is a bio-inspired protocol from bees' communication, basing on direction by using sun as a compass. The principle of BeeRP is illustrated in figure 5 where:

- Each sensor node is equipped with a compass.

- The transmitter is considered as a hive of bees.

- The receiver (sink in WSN) is considered as a sun

- The transmitter's neighbors that can communicate directly with this later are considered as sources of food.

The transmitter that will communicate indirectly with the receiver will use its own compass to select the smallest angle that will be limited between the straight edge (from transmitter to receiver) and edge from transmitter to one of its neighbors. The selected neighbor will do the same task by using its own compass to select the closest of its neighbors that creates a smallest angle to reach the receiver and so on till reaching the final target (receiver).

BeeRP which is a bio-inspired algorithm is the same as DIR or Compass routing in its advantages and insufficiencies.

The following algorithm describes Declivity in homogenious network (network without obstacles or holes)

\section{BeeRP-Algorithm}

Let $\mathbf{U}$ the current node (node that detects the event)

Let $\mathbf{A}$ array with length $=\mathrm{N}$, containing $\mathrm{N}$ neighbors (the set of nodes in communication range of the sink-node) ordered from the nearest to the farthest from $\mathrm{U}$ in terms of angle as it is explained previously $\{/ \mathrm{A}[1]=$ the smallest angle, $\mathrm{A}[2]=$ the following smallest angle, ........., A[N]=the latest angle) */\}

1. For each sensor node Do

2. If next-hop= sink-node Then

3. Exit $\{/ *$ Routing has succeeded $* /\}$

Else

4. Select $A[1]\{/ *$ Select the nearest neighbor that has the smallest angle toward the final destination (sink-node)*/\}

5. End If

\section{Example of Simulation of BeeRP \& DIR Protocols}

We have simulated our work using VisualSense 1.0.7, free download from[15] with the following characteristics: 
- Sensor-nodes are static, deployed in a surface of $1250 \times$ 1250 square, they are uniformly distributed over the area;

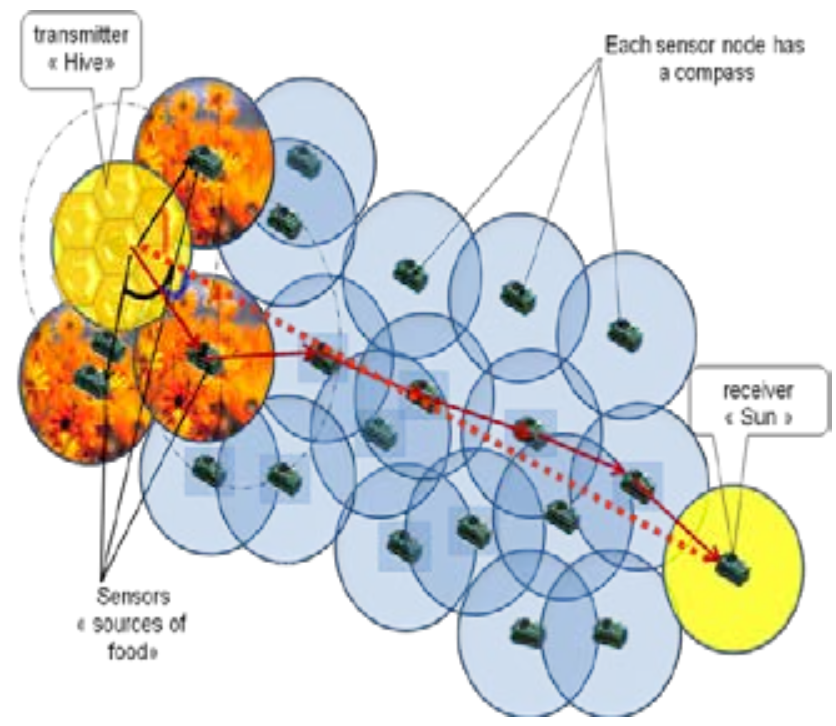

Figure 5. BeeRP: Bee Routing Protocol for WSN

- All nodes have the same maximum transmission range Rmax $=200$;

- The single fixed sink-node is placed in the center of the area with $\mathrm{R}=200$;

- Each sensor node will have two coordinates $(\mathrm{x}, \mathrm{y})$ as real coordinates given by the landmark of VisualSense, the sink node will have as coordinates $(0,0)$ representing the center of the network.

The two protocols BeeRP and DIR have been tested on a homogeneous network as it is illustrated in the following figure (figure 6).

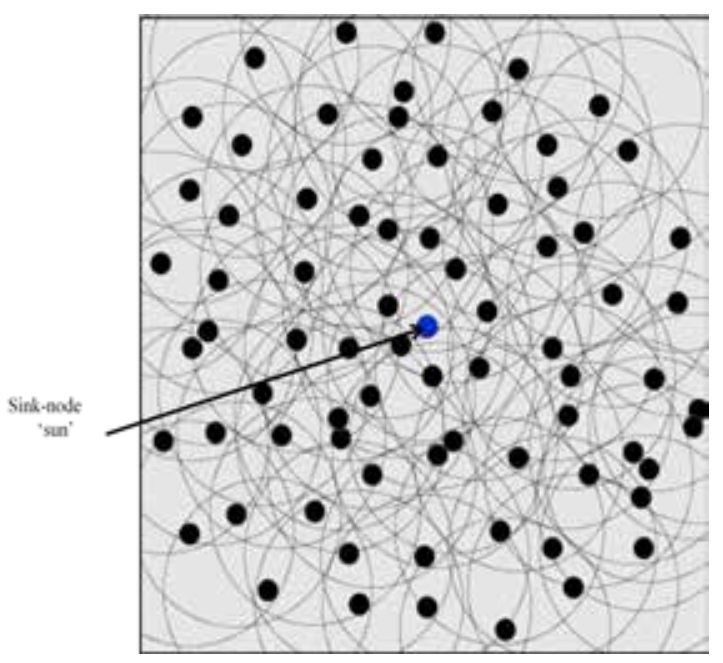

Figure 6. Homogeneous network

In a homogeneous network (either dense or not), BeeRP and DIR always succeed to guarantee delivery (see figure 7). The paths found represent the unique shortest paths existing in the network.

BeeRP and DIR follow the same paths as it is illustrated in the figures 7 and 8.

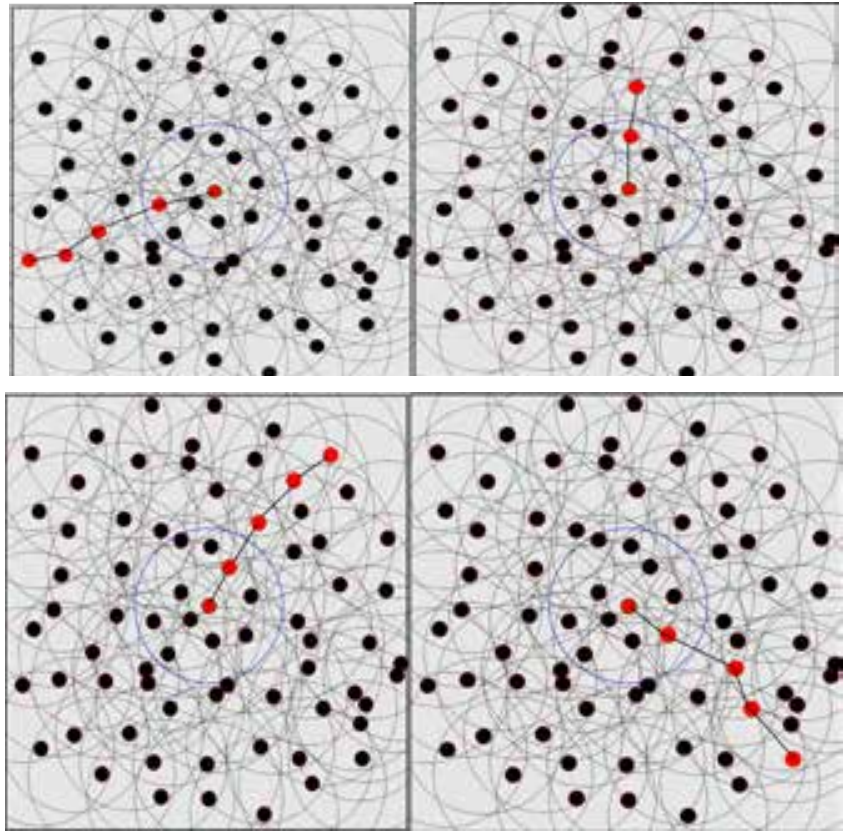

Figure 7. The same paths followed by BeeRP \& DIR.

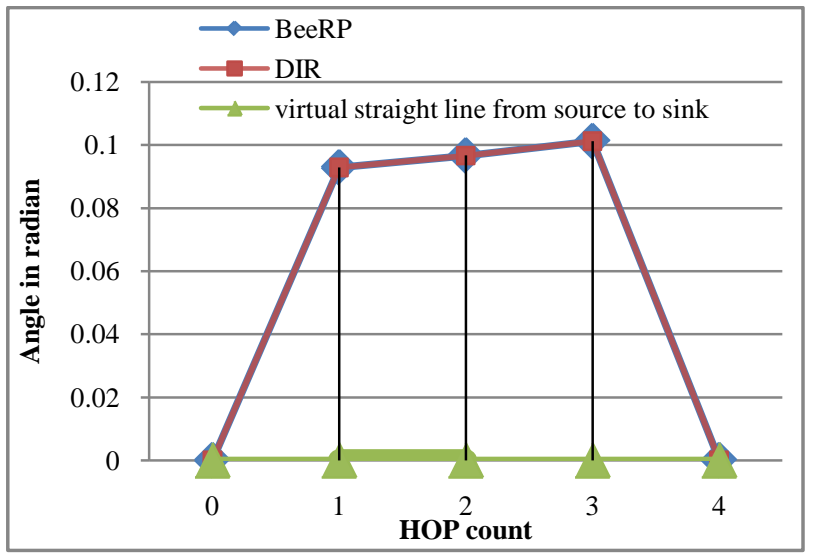

(a)

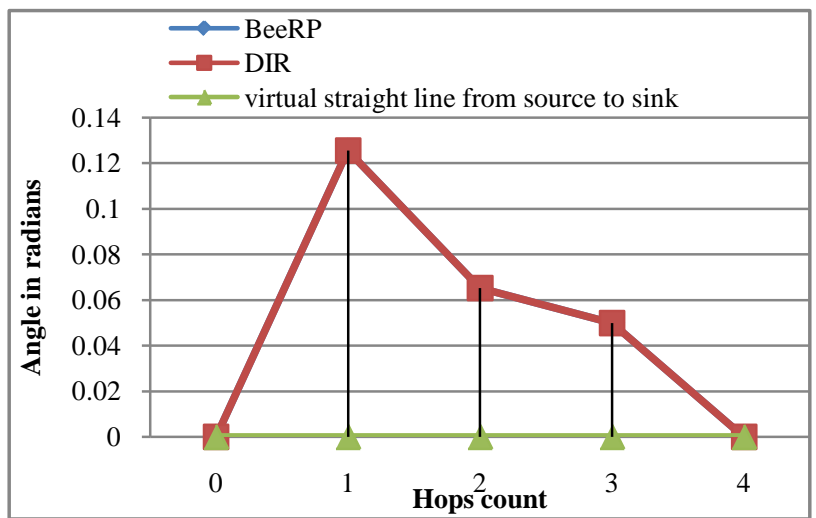

(b)

Figure 8. Example of comparison between BeeRP and DIR

\section{Conclusions}

The aim of any proposed routing protocol whether new or old and whatever its divergence and different research tools is to optimize the path from the transmitter to the receiver 
with a perfect economy of the sensor nodes' energy and a perfect maximization of the sensor networks' lifetime.

In the light of this fact, we have first tried to propose a bio-inspired routing algorithm, called BeeRP. It is based on natural bees' communication. It has proved to be efficient in either a homogenous or a dense network. Afterwards, this bio-inspired protocol is compared with another algorithm in the geometric field called DIR routing protocol. Through this comparison, we have found that both algorithms use the notion of angles to choose the shortest path by selecting the smallest angle in each hop toward the final destination. As a conclusion we have found that these two protocols are utterly and strikingly similar. Thus, the outcome of this paper is to show the relationship between the geometric field and the bio-inspired field. This relation proves that these two fields can be complementary.

As a perspective, we hope that some aspects will be analyzed such as the study of BeeRP towards asymmetric links and extension to heterogeneous networks.

\section{REFERENCES}

[1] Dhinu Johnson Kunnamkumarath, “A Model Driven Data Gathering Algorithm For Wireless Sensor Networks”, a Thesis submitted in partial fulfillment of the requirements for the degree MASTER OF SCIENCE, 2008.

[2] I. Akyildiz, W. Su, Y. Sankarasubramaniam, and E. Cayirci, "A survey on sensor networks" IEEE Communications Magazine, Volume 40, Issue 8, pp.102-114, August 2002.

[3] Farooq M, "From the Wisdom of the Hive to Intelligent Routing in Telecommunication Networks: A Step towards Intelligent Network Management through Natural Engineering”, PhD Thesis, University of Dortmund, Germany, 2006.

[4] H. F.Wedde, M. Farooq, Y. Zhang, "Beehive: An efficient fault tolerant routing algorithm inspired by honey bee behavior”, Ant Colony, Optimization, and Swarm Intelligence, Vol. 3172 of LNCS, Springer, pp. 83-94, 2004.
[5] Wedde HF, Timm C, Farooq M, BeeHiveGuard, “A Step Towards Secure Nature Inspired Routing Algorithms”. In Rothlauf et al., editors, Proocedings of the EvoWorkshops 2006, Lecture Notes in Computer Science, Budapest, 2006.

[6] Basil Etefia, "Routing Protocols for Wireless Sensor Networks”, Summer Undergraduate Program in Engineering at Berkeley, (SUPERB) 2004.

[7] S. Olariua, Q. Xu, A. Wadaa, I. Stojmenovic, "Virtual Infrastructure for Wireless Sensor Networks”, Book style, pp.107-137, 2005.

[8] J. von Neumann, "Probabilistic logics and the synthesis of reliable organisms from unreliable components", Automata Studies 34, PP. 43-99, 1956.

[9] J. Maynard Smith, "Evolution and the Theory of Games", Cambridge University Press, 1982.

[10] O. Babaoglu, G. Canright, A. Deutsch, G. A. Di Caro, F. Ducatelle, L. M. Gambardella, N. Ganguly, M. Jelasity, R. Montemanni, A. Montresor, T. Urnes, "Design patterns from biology for distributed computing”, ACM Trans. Auton. Adapt. Syst. 1 (1) (2006) 26-66. doi:10.1145/ 1152934.1152937.

[11] M. Meisel, V.Pappasb, L.Zhang, “A Taxonomy of Biologically Inspired Research in Computer Networking”, June 26, 2009.

[12] Von Frisch K, “The Dance Language and Orientation of Bees”. Harvard University Press, Cambridge, 1967.

[13] H. F. Wedde, M. Farooq, T. Pannenbaecker, B. Vogel, C. Mueller, J. Meth, R. Jeruschkat, BeeAdHoc: an energy e_cient routing algorithm for mobile ad hoc networks inspired by bee behavior, in: Proc. GECCO, ACM, 2005, pp. 153-160.

[14] E. Kranakis, H. Singh and J. Urrutia, Compass routing on geometric networks, Proc. 11th Canadian Conference on Computational Geometry, Vancouver, August, 1999.

[15] http://ptolemy.eecs.brekeley.edu 